\title{
Fitness Staff's Perceptions of Offering the 3-Step Workout for Life Program in Senior Living Communities
}

\author{
Maren Jorgensen \\ College of Public Health and Health Professions
}

Faculty Mentor: Chiung-ju Liu, Department of Occupational Therapy

\begin{abstract}
Functional exercise programs have been shown to improve activities of daily living and delay the disablement process in older adults. However, the majority of senior living communities do not utilize functional exercise within their fitness programming. The 3-Step Workout for Life program combines exercise and activities of daily life to improve independence and functional gains in older adults. This research study aims to understand the perceptions that senior fitness personnel have towards the 3-Step Workout for Life to determine the program's feasibility. Individual semi-structured interviews were conducted with five fitness instructors from communities in Florida that provide independent living options to senior residents. Interviews were audio-recorded, transcribed, and then analyzed using thematic analysis techniques on NVivo 12 to identify common themes. Four key themes emerged. Three themes suggested modifications to the program: 1) revise screening process; 2) adjust group resistance band exercise; 3) redesign individualized one-on-one ADL exercise. The fourth theme acknowledged that the 3-Step Workout for Life addresses a gap in senior fitness. The results of this study provided insight into the feasibility of this program and helped direct modifications needed to enable future integration.

Keywords: activities of daily living, community-based intervention, exercise
\end{abstract}

\section{Introduction}

It is projected that by 2030, one in five Americans will be 65 years or older (United States Census Bureau, 2019). Chronic diseases and age-related physical decline increase the risk of late-life disability (Fong, 2019), placing enormous demands on medical and therapeutic services. Specifically, age-related decline in muscle mass and strength along with reductions in physical activity, correlate with the progressive decline in function and loss of independence in older adults (Hirani et al., 2015). Although there have been many advancements in medical care that extend the life span, physical exercise remains the most universal and effective treatment to prevent disability in later life (Booth, Roberts, \& Laye, 2012).

The American College of Sports Medicine recommends regular physical exercise that includes aerobic, resistance, flexibility, and neuromotor exercise of older adults (Garber et al., 
2011). While physical exercise improves muscle strength, aerobic capacity, flexibility, and balance, it is less clear whether these traditional late-life exercise regimens effectively minimize physical disability (Keysor, 2003). Alternatively, functional exercise programs that integrate strength and balance activities into regular daily tasks have been shown to reduce functional limitations in older participants. Programs that combine traditional strength training and activities of daily living (ADLs), such as Lifestyle-Integrated Functional Exercise (Gibbs et al., 2015), ADL-based training (Dobek, White, \& Gunter, 2007), and the 3-Step Workout for Life (Liu, Donovan, \& Wolford, 2020) enable older adults to maintain independence and improve ADL performance.

Senior living communities (SLCs) are popular alternative housing arrangements the elderly (Mullen et al., 2007). Older adults relocate to SLCs due to family pressure, transportation issues, challenges in maintaining a home, and attraction to the supportive amenities that these facilities offer (Roy, Dubé, Després, Freitas, \& Légaré, 2018). SLCs are in the unique position of promoting and providing exercise resources including gyms, fitness personnel, and exercise classes for aging residents. Although many exercise programs have been tested in SLCs, few have been implemented.

The 3-Step Workout for Life program is an evidence-based task-oriented exercise program that combines exercise and occupational therapy principles to improve independence and functional gains in older adults. In a previous feasibility study of the 3-Step Workout for Life, participants showed significant improvements on the Box and Block Test and the 30-second Chair Stand Test, assessments that evaluate physical functioning of the upper and lower extremities, respectively. In measures that assess ADL performance, including the Late-Life Function and Disability Instrument and the Assessment of Motor and Process Skills, participants demonstrated marked improvements in function and motor skills. These results indicate that the 3-Step Workout for Life is an effective program in mitigating late-life disability (Liu, Jones, Formyduval, \& Clark, 2016). The 3-Step Workout for Life is a 10-week program that utilizes resistance and $\mathrm{ADL}$ exercise. In the resistance training portion of the program, an instructor will lead a group of four to six residents through elastic band exercises to improve muscle strength. In the ADL exercise stage, an instructor conducts one-on-one personalized exercise sessions in each resident's home. The participant's household chores and tasks are adapted for exercise purposes. Ideally, existing fitness personnel from the SLC would manage and deliver this program. This 
study aims to assess fitness staff's perceptions of offering the 3-Step Workout for Life program within their communities, laying the groundwork for future implementation.

\section{Methods}

\section{Research design}

A one-time survey study design using a semi-structured interview format was employed.

\section{Participants}

Participants were recruited from fitness departments in SLCs that included independent living residences. A participant was eligible if:

1. His or her job responsibilities included the direct delivery of exercise to senior residents

2. He or she worked in the community as a fitness staff member for at least six months

A participant was excluded if he or she exclusively provided single specialized exercise instruction or if two colleagues from the same community already participated in the study. The screening process to determine eligibility was conducted during the recruitment phone call or email.

\section{Recruitment plan and procedure}

A Google search was conducted to compile a list of SLCs that included residential fitness amenities. A list of 50 SLCs located in Gainesville, Ocala, Tampa, and Jacksonville was compiled between August and September 2020. Fitness staff were contacted via email or phone call and invited to participate in this study.

\section{Study procedure}

Telephone consent or electronic consent (eConsent) was conducted depending on the preference of the eligible participant between October and December 2020. The interview was conducted on the Zoom platform and a pre-determined list of questions was developed to guide the interview. The questionnaire investigated the participant's job responsibilities, the community's exercise facilities and programs, residents' participation in exercise, and their perceptions of the 3-Step Workout for Life program. The interview was audio-recorded and automatically transcribed by Zoom. The interviews lasted about one hour and the participant was compensated $\$ 20$ in the form of a gift card. 


\section{Data analysis}

Reviewed transcripts were uploaded to the computer program NVivo 12 for coding purposes. The transcription underwent two cycles of coding and thematic analysis by two researchers during January and February 2021. After the two researchers discussed their codes, common themes for each specific aim were identified.

\section{Results}

Five fitness providers recruited from different SLCs completed the interviews. Four participants held administrative positions. The participants were all female, self-identified as Caucasian, and had an age range from 40 to 60 years old. All participants had prior experience leading group fitness classes, ranging from 7 to 40 years of experience. Four key themes were identified from the thematic analysis. Subthemes in support of each key theme were further identified. Participant quotes are included below.

\section{Theme 1: Revise screening process}

The 3-Step Workout for Life program was designed to serve older residents experiencing frailty and ADL limitations. A cognitive and physical screening is performed to enroll residents in the program. This approach clashes with the typical first-come, first-serve nature of SLC's fitness classes and gym amenities. Although not included in their current job responsibilities, participants were open to conducting screenings. However, three participants suggested modifying the screening process. Two sub-themes emerged: offer program alternatives and present a positive outlook.

Offer alternatives. If a resident is labeled ineligible, it can adversely affect their self-esteem. By offering alternatives to residents that do not qualify for the program, fitness personnel would encourage residents to exercise at an appropriate level.

“... [to resident] your assessment shows that you have a good level of functional capacity, so you're not really eligible for this program and then direct them somewhere else."

Positive outlook. Participants discussed the importance of maintaining a positive outlook when conducting the screening. It was acknowledged that mental and physical assessment can be disheartening for older residents. The screening process should emphasize the residents' capabilities, not focus on their deficits. 
"I would be very careful and be very sensitive how you word it. And make it positive, always positive. Because you have to realize that the people that you're dealing with, at this point in their life, are dealing with so many negatives. ...if you're going to categorize them, make it as positive and upbeat as you possibly can.”

\section{Theme 2: Adjust group resistance band exercise}

The 3-Step Workout for Life program utilizes resistance band exercise to improve muscle strength. An instructor leads exercises, teaching four to six residents at a time. When considering the feasibility of this stage of the program, two subthemes emerged: resistance exercise is familiar but the class size is unrealistic.

Resistance band exercise is familiar. The participants noted that resistance band exercises are commonly used in their facilities' group fitness classes and they were experienced instructing resistance band exercise.

"Lots of experience. That's one of our number one pieces of equipment."

"We do resistance band training. I always use them in my balance class so that's three days a week. ...we already incorporate a lot of that in the classes that we currently teach."

Class size is unrealistic. Three participants noted that a small class size is appropriate as more supervision would reduce injury and allow instructors to provide individualized attention.

"If you're working with only four or six people, you have the opportunity to give some very specific direction...versus a class."

However, four participants noted that the small class sizes would need to be expanded to prevent cost from being prohibitive.

"I think that our instructors can handle the average 15-person class and still correct form."

"The cost of paying an instructor for an hour to supervise four to six people...you won't make the money back to pay for the class. Group activities are very expensive."

\section{Theme 3: Redesign individualized one-on-one ADL exercise}

Participants' responses suggested that their communities' existing fitness infrastructure could not support a one-on-one instruction format. Despite interest in incorporating daily living activities as exercise, the ADL portion of the 3-Step Workout for Life program would require 
extensive modification for implementation. Four subthemes emerged: conflicted with job responsibilities, additional staff needed, structural adaptation required, and format ill-suited to resident lifestyle.

Conflicted with job responsibilities. All participants noted that their responsibilities, and those of existing fitness personnel, conflicted with the time needed to complete this stage of the program.

"The ADL component...I would not be able to do that. It's not feasible for one person."

"My full-time personal trainers also have supervisory responsibilities in the fitness center so they really can't leave to go into residents' homes."

Additional staff needed. Four participants noted that additional staff or external contractors would be required to conduct this program, demanding additional financial resources.

"You [could] use an independent contractor who is trained in your program and wants to do the initial startup with somebody."

"The next thing that comes to mind is compensation. Our instructors, our personal trainers make anywhere from $\$ 20$ to $\$ 25$ a session. So, are we factoring cost into this to compensate the personal trainer?"

Three participants mentioned that they would not feel qualified conducting ADL, one-onone exercise; and two suggested that their community's rehabilitative staff lead these exercises.

"One of the things I talk about is staying within your scope. And I would not feel comfortable going and talking about those types of things specific to ADL functioning because that's the job of an occupational therapist."

Structural adaptation required. The structure of individualized ADL exercise also presented an obstacle to program feasibility. The participants agreed that the timeline for the program is conducive to providing residents with measurable outcomes and improvements. However, two acknowledged that a program occurring biweekly, rather than three times a week, would be more tenable for fitness staff and likely increase resident compliance and enrollment.

"Two days a week program is the easiest for us to work with because of their [resident's] existing schedule in the community, they have things to do. But two days a week ... seems to be a good number of class-type activities for a person to engage in." 
Two participants voiced concerns with home-based instruction, due to safety concerns. None of the facilities surveyed offer individualized, in-home fitness instruction.

"They're [fitness staff] in a situation with somebody pretty frail and they're in their home, and that they don't have any backup or support in that environment, should anything unusual occur."

Ill-suited to resident lifestyle. Participants provided conflicting responses regarding residents' perceptions towards this portion of the program. Participants acknowledged that residents would prefer at-home fitness programming, to retain privacy and to better adhere to the program's demands. However, two participants noted that residents may be unwilling to allow fitness staff to enter their homes.

"We have a lot of residents here that don't come to the facility because either they don't feel comfortable or they need something to help them with their strength and mobility. I do think it's a great idea and I would think people would be interested."

"I don't know if people would be open to having somebody come in their home."

Three participants agreed that the social aspect of exercise is a key motivator for older adults to engage in physical activity. An individualized program may be isolating or boring for residents.

"A lot of them deal with depression and loneliness at this point in their life because a lot of them are living alone. Encouraging them to come and be a part of the class or to find a buddy to walk with, those are the key things I think that are really important."

All of the participants mentioned that the large time demand could detract residents from enrolling and completing the program.

“... an hour can seem dauntingly long for that age group because they can only take so much at a time. ... if they're frail and they're in that category, you start passing that 30-minute mark, you're going to be pushing into shut down. I think an hour's a lot."

\section{Theme 4: Addresses a gap in senior fitness}

Despite a dedicated fitness staff and a wide range of fitness activities, residents in active communities still contend with age-related decline. Three subthemes emerged: frail residents remain a reality, potential benefit to residents, and lack of ADL-based exercise programs. 
Frail residents remain a reality. Two participants remarked that residents experiencing ADL impairment were less likely to seek fitness resources.

"We do have some of the aging population as well and those are the ones at home that no one ever sees. They are only playing cards or they do very minimal activity."

Potential benefit to residents. All of the participants agreed that residents struggling with frailty would benefit from exercise that incorporated ADL.

"... if they start out with struggling with some of those ADL and by the end of the program, they feel much more confident and competent, that's huge. That's what you want to see, you want them to experience that change for the better in themselves. Where if they're just coming to an exercise class every day, they might go up with a heavier weight or a stronger band. But if you're doing the same exercises every day, you're really just going to adapt to that exercise."

Lack of ADL-based exercise programs. Three participants incorporate daily living movements within their classes or explain the functional purposes of specific exercises. However, none of the communities utilized specific, one-on-one ADL exercise within their existing fitness programming, despite acknowledging its benefits.

"I like to incorporate rotation across body exercises ...we teach them why it is important... you're going to use it in about everything you do."

\section{Discussion}

This paper aimed to evaluate the perceptions of the 3-Step Workout for Life program from fitness staff working in SLCs. The current study built on a prior small implementation study of the 3-Step Workout for Life program in a local SLC (Liu, Donovan, \& Wolford, 2020). This is the first study that systematically evaluated fitness staff's perceptions of this program. Data analysis yielded four overarching themes that addressed program impediments.

The first theme, revise screening process, revealed that fitness staff are willing to conduct resident screening if the procedure offered alternatives and maintained a positive outlook. Although communities' fitness amenities and resources are open to all residents, the majority of participants had previous experience conducting fitness assessments and/or gym orientations. Concerns were limited to the screening process itself because it may disenfranchise the very 
clients who stand to benefit the most from increasing their physical activity. By offering alternatives to ineligible residents, fitness personnel would continue to encourage their residents to exercise. The screening process should be designed to help residents find the fitness programming that best addresses their functional needs.

The themes adjust group resistance band exercise and redesign individualized one-on-one $A D L$ exercise, indicated that the two exercise stages of the program required disparate levels of modification to increase feasibility. Because the resistance band exercise portion of the program mirrors the format of a typical group fitness class, fitness staff are not required to dedicate time or training for successful implementation. The main concern that arose was accommodating a small class size.

The ADL portion of the program, however, was identified as a significant departure from the participants' job responsibilities and preferred class structure. Although participants acknowledged the benefits associated with a functional-based fitness program, the time commitment required to implement this program was insurmountable. Participants indicated that they would need additional staff to make this stage of the program feasible, requiring greater financial support. Concerns were raised regarding the qualification necessary to conduct ADL exercise. It was recommended that more appropriate providers, including existent rehabilitative staff or external contractors, lead this portion of the program. Participants suggested extensive structural revisions to increase feasibility and raise their interest in delivering the program.

When inquiring about residents' perceptions towards ADL exercise, the participants provided a variety of answers. A few participants felt that residents would prefer at-home fitness programming to retain privacy and increase program adherence. However, some participants disagreed, noting that residents may feel uncomfortable having fitness staff in their homes. Some participants mentioned that socialization is a motivator to remain active for a population that often struggles with loneliness and isolation. Overall, the participants did not provide a comprehensive picture of how receptive residents would be to in-home ADL exercises. These responses may accurately reflect the range of perceptions residents would have towards the 3Step Workout for Life program.

Lastly, the theme addresses a gap in senior fitness, revealed that fitness staff are aware of the divide between fitness improvement and functional improvement. The communities and their staff have clearly invested a great deal of money, time, and resources to encourage residents to 
be active. However, residents, even those who utilize fitness amenities, may still struggle to complete daily living tasks independently. The participants recognized that ADL-based exercise would beneficially impact their residents' health outcomes. Currently, specialized ADL instruction is costly, solely provided by rehabilitative therapists, and only addresses the needs of residents recovering from an injury or those requiring a high level of care (e.g. assisted living).

Participants found the 3-Step Workout for Life program to be a promising, novel way to combat age-related decline in community-dwelling adults. The modifications to the first half of the program are easily accommodated; however, the recommended changes to the ADL aspect of the program would likely nullify the benefits residents would experience. Fitness personnel were unable to offer the program through the existing infrastructure, due to the time demand, financial compensation, or home-based component. Instead, the program could be offered through different channels within the community. It may be more effective to utilize available life-long learning infrastructure within the facility or involve therapeutic staff to deliver the program.

This study has several limitations. First, the participants were recruited through non-random sampling methods, which may have resulted in a non-representative sample of the general population. The participants who enrolled in this study had similar demographic information; it is likely that the perspective gained from a more diverse sample would provide a greater variety of responses. Additionally, the small sample size impacts the scope of this study's findings. Recruitment was conducted during the COVID-19 pandemic. In many SLCs, fitness departments were closed to residents. As a result, it was challenging to contact fitness personnel. In many less affluent communities, the fitness departments were disbanded. For this reason, the participants recruited in this study represented more affluent faculties, where limited fitness programming services were maintained. Therefore, the responses may not be representative of the resources available at less affluent facilities.

Despite these limitations, this study is a step toward understanding the aspects of the 3-Step Workout for Life program that require revision for future implementation in SLCs. By consulting those potentially delivering the program, barriers within the existing SLC infrastructure were identified and modifications were proposed to improve integration. Future research should seek to implement the modifications suggested by the participants in this study. 


\section{Acknowledgements}

This work was supported by the University Scholars Program within the Centers for Undergraduate Research at the University of Florida. The author wishes to acknowledge the invaluable assistance of Lorraine Penias and Angela Cao with participant recruitment.

\section{References}

Booth, F. W., Roberts, C. K., \& Laye, M. J. (2012). Lack of Exercise Is a Major Cause of Chronic Diseases. Comprehensive Physiology. doi: 10.1002/cphy.c110025

Dobek, J. C., White, K. N., \& Gunter, K. B. (2007). The Effect of a Novel ADL-Based Training Program on Performance of Activities of Daily Living and Physical Fitness. Journal of Aging and Physical Activity, 15(1), 13-25. doi:10.1123/japa.15.1.13

Fong, J. H. (2019). Disability Incidence and Functional Decline among Older Adults with Major Chronic Diseases. SSRN Electronic Journal. doi:10.2139/ssrn.3421968

Garber, C. E., Blissmer, B., Deschenes, M. R., Franklin, B. A., Lamonte, M. J., Lee, I., . . Swain, D. P. (2011). Quantity and Quality of Exercise for Developing and Maintaining Cardiorespiratory, Musculoskeletal, and Neuromotor Fitness in Apparently Healthy Adults. Medicine \& Science in Sports \& Exercise, 43(7), 1334-1359. doi:10.1249/mss.0b013e318213fefb

Gibbs, J. C., Mcarthur, C., Milligan, J., Clemson, L., Lee, L., Boscart, V. M., . . Giangregorio, L. M. (2015). Measuring the implementation of a group-based Lifestyle-Integrated Functional Exercise (Mi-LiFE) intervention delivered in primary care for older adults aged 75 years or older: A pilot feasibility study protocol. Pilot and Feasibility Studies, 1(1). doi:10.1186/s40814-015-0016-0

Hirani, V., Blyth, F., Naganathan, V., Couteur, D. G., Seibel, M. J., Waite, L. M., . . Cumming, R. G. (2015). Sarcopenia Is Associated With Incident Disability, Institutionalization, and Mortality in Community-Dwelling Older Men: The Concord Health and Ageing in Men Project. Journal of the American Medical Directors Association, 16(7), 607-613. doi:10.1016/j.jamda.2015.02.006

Keysor, J. (2003). Does late-life physical activity or exercise prevent or minimize disablement? A critical review of the scientific evidence. American Journal of Preventive Medicine, 25(3), 129-136. doi:10.1016/s0749-3797(03)00176-4

Liu, C., Donovan, J., \& Wolford, C. L. (2020). Feasibility of staff-led 3-Step Workout for Life to reduce late-life activities of daily living disability. International Journal of Rehabilitation Research, 43(2), 141-147. doi:10.1097/mrr.0000000000000396

Liu, C., Jones, L. Y., Formyduval, A. R., \& Clark, D. O. (2016). Task-Oriented Exercise to Reduce Activities of Daily Living Disability in Vulnerable Older Adults: A Feasibility Study of the 3Step Workout for Life. Journal of Aging and Physical Activity, 24(3), 384- 392. doi:10.1123/japa.2015-0070

Mullen AJ, Singer H, ProMatura Group, National Investment Center for the Seniors Housing \& Care Industries (2007). NIC National Housing Survey of Adults age 55+: Opinions, Attitudes, Perceptions and Behaviors. Annapolis, MD: National Investment Center for the Seniors Housing $\&$ Care Industry. 
Roy, N., Dubé, R., Després, C., Freitas, A., \& Légaré, F. (2018). Choosing between staying at home or moving: A systematic review of factors influencing housing decisions among frail older adults. Plos One, 13(1). doi:10.1371/journal.pone.0189266

US Census Bureau. (2019, October 10). Older people projected to outnumber children. Retrieved from https://www.census.gov/newsroom/press-releases/2018/cb18-41-population- projections.html 